\title{
Cost-effectiveness analysis comparing robotic sacrocolpopexy to a vaginal mesh hysteropexy for treatment of uterovaginal prolapse
}

\author{
Patrick J. Culligan*, Charbel Salamon, Christa Lewis, Troy D. Abell ${ }^{\#}$ \\ Atlantic Health System Division of Urogynecology and Reconstructive Pelvic Surgery, Morristown, USA \\ Email: *patrick.culligan@atlantichealth.org
}

Received 23 July 2013; revised 21 August 2013; accepted 30 August 2013

Copyright (C) 2013 Patrick J. Culligan et al. This is an open access article distributed under the Creative Commons Attribution License, which permits unrestricted use, distribution, and reproduction in any medium, provided the original work is properly cited.

\begin{abstract}
Objective: To compare costs and QoL associated with 2 minimally invasive operations to treat uterovaginal prolapse. Study Design: A decision analytic cost-effectiveness model comparing vaginal mesh hysteropexy to robotic-assisted sacrocolpopexy. Costs were derived from a hospital perspective. QoL estimates focused on: recurrent prolapse; erosion; infection; transfusion; cystotomy; chronic pain; lower urinary tract symptoms; and mortality. Actual procedural costs at our institution were calculated. Costs and quality adjusted life years were examined over 1 year. Results: The costs $(\$ 21,853)$ and QALYs $(0.9645)$ for robotic sacrocolpopexy produced a CE Ratio of $\$ 22,657$ per QALY. The costs $(\$ 14,890)$ and QALYs (0.9309) for vaginal mesh produced a CE Ratio of $\$ 15,995$ per QALY. The incremental cost per QALYs for robotic surgery was $\$ \mathbf{2 0 7 , 2 3 2}$. Sensitivity analysis on all utilities, cost estimates, and complication estimates didn't cross any thresholds. Conclusion: Vaginal mesh was more cost-effective than robotic sacrocolpopexy even when the cost of the robot was not factored.
\end{abstract}

Keywords: Cost-Effectiveness; Robotic-Sacrocolpopexy; Uterovaginal Prolapse; Vaginal-Mesh

\section{INTRODUCTION}

Minimally invasive surgical approaches for the treatment of pelvic organ prolapse are becoming quite common, and transvaginal mesh placement in particular has become increasingly popular. Of the nearly 200,000 prolapse operations performed annually in the U.S., one third involve synthetic mesh, with three fourths of these

\footnotetext{
"Corresponding author.

"Troy Abell did his work at Baylor University.
}

mesh repairs being performed transvaginally [1]. The "kits" used for transvaginal mesh placement have evolved rapidly-creating difficulty for anyone wanting to conduct traditional surgical trials comparing these minimally invasive techniques. Researchers using traditional study methods run the risk of their specific study-surgeries being obsolete before their study is finished. Moreover, traditional research studies typically determine superiority or non-inferiority without considering costs.

Decision analytic studies - on the other hand - are useful in this rapidly changing environment, because they allow for the consideration of specific health outcomes in terms of economic costs and quality of life simultaneously. Decision analytic studies are designed to help clinicians determine the best clinical course of action in situations where there is no obvious superior choice. These studies are best viewed as a way to analyze clinical decisions without having to spend the time and money required to do traditional studies, such as RCT's. When constructing any decision analytic model, the researchers start with a very specific research question. This research question should represent a clinical choice between two clinical courses of action-ideally a choice that is commonly made by clinicians every day. After deciding on the clinical question to be asked, the decision tree is built to take into account all relevant positive and negative ramifications of either clinical course of action. Common endpoints used for decision analysis studies include "long-term survival"; "quality of life" scores; societal or hospital costs; or a combination of all of these-called "quality adjusted life years" (QALY). Thus decision analytic studies are meant to aid clinicians as they make decisions about patient care - not to replace traditional research. One now-famous example of this study design was published in 2005 by Parker et al. 
promoting the idea of ovarian conservation at the time of hysterectomy for benign disease [2].

With the unique benefits of this research design in mind, we applied decision analytic study techniques to a clinical scenario that commonly happens in our practice - deciding between two rather different surgical approaches for a subset of patients suffering from utero-vaginal prolapse. The two procedures in question were a transvaginal mesh hysteropexy "kit" (Uphold ${ }^{\mathrm{TM}}$ vaginal support system, Boston Scientific, Natick, MA) and a robotic-assisted laparoscopic supracervical hysterectomy and sacrocolpopexy (Intuitive Surgical, Sunnyvale, CA).

\section{MATERIALS AND METHODS}

Following Atlantic Health System IRB approval, we developed a decision analytic model using commercially available software (TreeAge Inc., Williamstown MA). Our model was designed to compare the cost effectiveness of robotic sacrocolpopexy to the Uphold ${ }^{\mathrm{TM}}$ (Boston Scientific, Natick, MA) vaginal mesh hysteropexy for treatment of uterovaginal prolapse. The choice between these two surgical options defined the two main branches of the decision tree. In other words, the primary decision evaluated in our model was whether to perform one or the other of these two surgical options on a patient. The subsequent branches of the tree were built to reflect the relevant good and bad clinical outcomes associated with either procedure. As the complete tree structure was too large for inclusion in this manuscript, a schematic version is shown as Figure 1. The timeframe we considered was the surgical procedure plus 12 months post-operative follow-up - including subsequent treatment of complications or recurrent prolapse. We chose this time frame for two reasons: 1) a large proportion of POP surgery failures happen within the first 12 post-operative months and 2) consideration of 12 months simplified our cost analysis within the model $[3,4]$.

The clinical description and demographics of an aver-

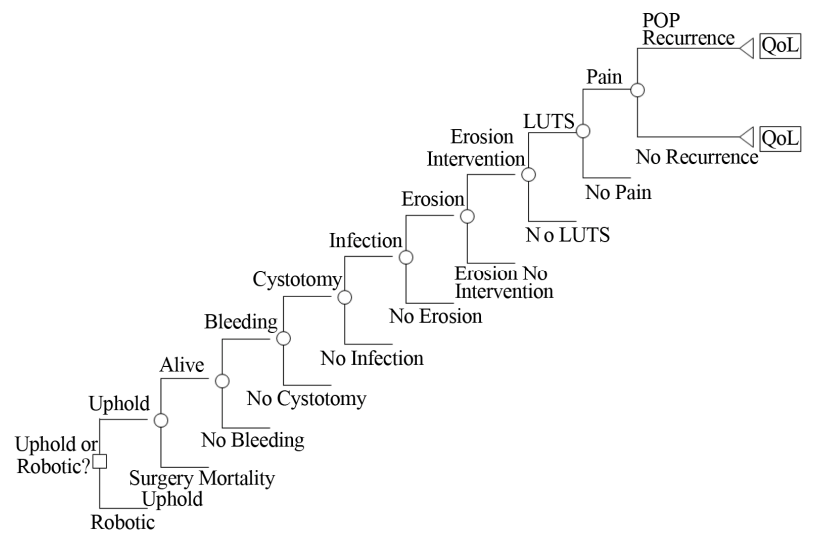

Figure 1. "Skeletal" view of decision tree. age patient were derived from a small group of actual patients in our practice. To identify these patients, we reviewed the charts of all patients in our practice that underwent the Uphold ${ }^{\mathrm{TM}}$ procedure in 2009. From that group we identified a subset of patients who were offered a choice between the Uphold ${ }^{\mathrm{TM}}$ procedure and a roboticassisted laparoscopic supracervical hysterectomy plus sacrocolpopexy. We identified 16 such patients. During the informed consent process for all 16 patients, (each carried out by the lead author-PC) the patients were provided with information regarding the risks, benefits, alternatives and post-operative restrictions associated with each operation in as objective a way as possible. All of these patients were also offered traditional native tissue repairs. In each case, the attending physician believed that any of the surgical options would be a good clinical choice and that the final decision was simply up to the patient. Interestingly, of the 16 patients who were offered that choice, 8 chose Uphold $^{\mathrm{TM}}$ and 8 chose robotic surgery. For the purposes of the study, we determined the mean and median clinical and demographic characteristics of these 16 patientssimply to give readers a better clinical reference point as they contemplate our results. Information from these 16 patients' hospital records were also used to derive the cost estimates used in the decision tree.

The major complications (of both surgical options) considered crucial to this analysis were: 1) blood loss requiring blood transfusion; 2) cystotomy; 3) surgical site infection; 4) vaginal mesh exposure managed in an office setting; 5) vaginal mesh exposure requiring surgical intervention; 6) re-operation for prolapse; 7) lower urinary tract symptoms (LUTS); 8) de novo chronic pain; and, 9) surgical mortality.

Probabilities of each clinical consequence (i.e. complications as well as positive outcomes) were derived from the medical literature whenever possible (Table 1) and were entered into the model as dichotomous branch points or "nodes". These estimates were derived from a systematic review of each topic-keeping in mind the scope of the decision tree model. When no relevant probabilities for a given clinical scenario could be found in the published medical literature, the authors used their clinical experience to estimate the values in question.

For the purposes of the model, we assumed the following when determining quality of life (QoL) and cost estimates: 1) blood transfusions would occur without complications, 2) cystotomies would be recognized and successfully repaired at the time of surgery; 3) surgical site infections would be successfully managed; 4) any vaginal mesh exposures-managed either in the office or operating room setting would heal well with no further sequellae; 5) all patients with recurrent pelvic organ prolapse would undergo re-operation; 6) LUTS would be 
Table 1. Estimates and sensitivity analysis for probabilities of adverse clinical outcomes.

\begin{tabular}{|c|c|c|c|c|c|c|}
\hline Adverse Clinical Outcomes & $\begin{array}{c}\text { Prevalence Estimates } \\
\text { Vaginal Mesh }\end{array}$ & $\begin{array}{c}\text { Prevalence Estimates } \\
\text { Robotic }\end{array}$ & References & $\begin{array}{l}\text { Analysis } \\
\text { Range }^{*}\end{array}$ & $\begin{array}{c}\text { Effectiveness } \\
\text { Threshold Value }\end{array}$ & $\begin{array}{c}\text { CE Threshold } \\
\text { Value }\end{array}$ \\
\hline Surgical Mortality & 0.0096 & 0.0096 & {$[5,6,7]$} & $0.00001-0.10$ & 0.03541 (RSC) & None \\
\hline Bleeding (Transfusion) & 0.003 & 0.005 & {$\left[8,{ }^{* *}\right]$} & $0.001-0.99$ & None & None \\
\hline Cystotomy & 0.021 & 0.013 & {$[9,10]$} & $0.01-0.99$ & 0.746 (RSC) & None \\
\hline Surgical Site Infection & 0.005 & $0.005^{*}$ & {$\left[{ }^{* *}\right]$} & $0.001-0.99$ & 0.2666 (RSC) & 0.51 (Vaginal Mesh) \\
\hline Mesh Exposure (Office) & 0.11 & 0.005 & {$[11,12]$} & $0.001-0.99$ & 0.2490 (RSC) & None \\
\hline Mesh Exposure (Surgery) & 0.03 & 0.001 & {$\left[11,{ }^{* *}\right]$} & $0.001-0.99$ & None & None \\
\hline de novo LUTS ${ }^{* * *}$ & 0.1 & 0.0507 & {$[13,14]$} & $0.03-0.70$ & 0.15 (RSC) & None \\
\hline de novo Chronic Pain & 0.03 & 0.043 & {$[11,14]$} & $0.001-0.99$ & None & None \\
\hline Reoperation for Prolapse & 0.096 & 0.06 & {$[13,14]$} & $0.03-0.70$ & None & None \\
\hline
\end{tabular}

${ }^{*}$ Same wide range is used for both procedures in sensitivity analysis. ${ }^{* *}$ No specific estimates for this scenario. Therefore, this probability was estimated by Senior Authors (PC \& CS). ${ }^{* * *}$ Lower Urinary Tract Symptoms. RSC-Robotic Sacrocolpopexy.

persistent; and 7) de novo chronic pain would be persistent. Each of these assumptions were made because doing so made the decision tree more manageable without limiting our ability to consider the most important clinical consequences.

The three possible outcomes related to mesh exposure: 1) "no exposure", 2) "exposure managed in the office", and 3) "exposure requiring surgical intervention" were modeled in the decision tree as a series of binary outcomes-i.e. "exposure" versus "no exposure" and "exposure managed in the office" versus "exposure requiring surgical intervention".

Three complications - namely LUTS, de novo chronic pain, and re-operation for prolapse - posed a particular challenge with respect to creating the model. The estimates in the medical literature for the probability (or prevalence) of these outcomes failed to address the issue of joint probability (i.e. overlap) between two or more possible outcomes. For example, among the vaginal mesh patients, the baseline estimate for the probability of LUTS and de novo chronic pain were 0.10 and 0.03 respectively. We hypothesized that all of patients who would experience de novo chronic pain would also experience LUTS. That assumption did not change the overall estimate for the total prevalence of LUTS at 0.10 . We further assumed that among LUTS sufferers 0.30 of those patients would also have de novo chronic pain.

In addition, looking specifically at overlapping complications in patients undergoing vaginal mesh placement resulted in the following scenarios: $3 \%$ of the patients having both LUTS and de novo chronic pain; 7\% having LUTS and no de novo chronic pain; $0 \%$ having no LUTS accompanied by de novo chronic pain; and, $90 \%$ of patients having no LUTS or de novo chronic pain. The hypothesis that almost $100 \%$ of patients who developed mesh-related chronic pain would also experience LUTS was also evaluated by sensitivity analysis.

The issue of overlapping complications (or joint probabilities) came further into play when we considered the outcome of re-operation for prolapse along with LUTS and de novo chronic pain. We assigned a probability estimate of 0.06 to "re-operation for prolapse" among robotic sacrocolpopexy patients, and we made certain estimates (based on our clinical experience) regarding the joint probabilities for the other two outcomes. Specifically among patients going through re-operations for prolapse, we estimated the prevalence of "LUTS plus de novo chronic pain" and "LUTS only" to be $10 \%$ each, leaving $80 \%$ of re-operation patients estimated to have neither.

Costs for the two surgical approaches were derived from a hospital perspective, using the actual costs for the select group of 16 patients (mentioned above) who underwent either a robotic sacrocolpopexy or the Uphold ${ }^{\mathrm{TM}}$ procedure at Morristown Medical Center in 2009. All 16 patients had received identical pre and post-operative care, and they were all discharged on the morning after surgery. These facts led us to simplify our cost assessments for the two groups. We only considered costs that were directly tied to the operations themselves. In other words, we did not consider costs of pre-operative or post-operative care, because they were identical for the two groups. We only included actual direct operational costs of each procedure -leaving out the indirect costs such as depreciation of the daVinci robot itself. Therefore, the costs included in the model were for equipment or materials used during the surgery; actual payments to the surgeons and anesthesiologists; and actual salary costs of the operating room personnel. All costs were determined in 2009 U.S. dollars using an itemized approach incorporating all actual costs 
associated with the surgical procedures in question-from the hospital perspective only.

When determining "quality of life" values for a model like ours, researchers can get their information from the patients themselves or from an expert panel. Deriving these scores from actual patients in a standardized nonbiased and validated way is highly complicated and extremely expensive, so we selected a panel of health care providers and lay-women who assigned quality of life scores (also known as "utility scores") used in our model. The members of this expert panel were all female and the health care providers each had extensive experience in dealing with patients suffering from pelvic organ prolapse. The panel included 4 urogynecology nurses, 1 woman's health nurse practitioner, $1 \mathrm{Ob} / \mathrm{Gyn}$ resident, 1 urogynecology fellow, and 2 medical students. This panel met as a group to assign utility scores for each possible clinical outcome in the decision tree. These panel discussions were led by our senior author (TA) - a decision scientist with decades of experience leading similar groups in an impartial way. In this context, the outcome "successful surgical repair with no complications" was assigned a utility score of 1.0 - indicating $100 \%$ quality of life, and the outcome "mortality" was assigned a score of 0.0 indicating $0 \%$ quality of life. All of the potential complications, as well as key combinations of outcomes, were then assigned scores between 1.0 and 0.0 (Table 2). These quality of life estimates were multiplied by 1 to produce quality-adjusted-life-years (QALYs) for a one-year time frame. Given that both costs and QALYs focused only on the first post-operative year, the estimates were not discounted over time.

Table 2. Expert panel quality of life (QoL) estimates.

\begin{tabular}{lc}
\hline \multicolumn{1}{c}{ Possible Outcome } & QoL Estimate \\
\hline No Side Effects & 1.00 \\
Bleeding Requiring Transfusion & 0.99 \\
Cystotomy & 0.95 \\
Mesh exposure (Office Management) & 0.90 \\
Surgical Site Infection & 0.86 \\
Mesh exposure (Surgical Management) & 0.85 \\
Reoperation for Prolapse & 0.75 \\
de novo LUTS* & 0.66 \\
$\begin{array}{l}\text { de novo Chronic Pain } \\
\text { Combination of: } \\
\text { Reoperation for prolapse } \\
\text { - de novo LUTS } \\
\text { Surgical Mortality }\end{array}$ & 0.66 \\
\hline
\end{tabular}

*Lower urinary tract symptoms.
Once all of the cost, QoL, and probability of occurrence estimates were included in the model, the software was used to determine the average cost and the average effectiveness for both robotic sacrocolpopexy and the vaginal mesh "kit." The average cost was divided by the average effectiveness to provide the cost-effectiveness ratio (CE Ratio). The a) difference between the costs of the two procedures was divided by the b) difference in the effectiveness of the two procedures to estimate the c) incremental CE Ratio.

Sensitivity analyses were performed to assess the robustness of the model. The value of each cost, utility (effectiveness), and probability estimate was varied within a wide range of values to determine if there was a point at which the preferred strategy changed. Every cost and probability estimate in the model was subjected to this sensitivity analysis in an effort to determine whether "weak links" existed in the set of assumptions. These oneway analyses were performed by individually (i.e. one-ata-time) varying probability estimate and cost estimate in the model through a very wide "plausible range" to determine the robustness of the model. Throughout these plausible ranges of values, any value that created a reversal in the final decision of the model was called a "threshold value". For any such "threshold value" produced we determined whether that value fell within a clinically plausible range. We also performed select combinations of two-way and three-way sensitivity analysis estimates (i.e. varying two or three values within the model simultaneously) in an effort to discover any thresholds within the model.

\section{RESULTS}

The average direct operating room specific costs of the Uphold $^{\mathrm{TM}}$ procedure and the robotic supracervical hysterectomy/sacrocolpopexy were $\$ 14,890$ and $\$ 21,853$ respectively. Table 3 provides a list of various procedural costs for both types of surgery. The average effectiveness (QALYs) for the Uphold ${ }^{\mathrm{TM}}$ procedure and the robotic procedure were 0.9309 and 0.9645 respectively. The cost per QALY of the Uphold ${ }^{\mathrm{TM}}$ procedure was $\$ 15,995$ compared to a cost per QALY of $\$ 22,657$ for the robotic procedure. Therefore, the robotic surgery was estimated to produce higher quality of life, but at higher costs. The difference in quality of life between the two operations known as the "incremental QALYs" was 0.0366, and that difference was gained at an additional cost of $\$ 6963$.

The incremental CE ratio was $\$ 207,232$ - meaning that the incremental gain in quality of life derived (i.e. 0.0366) from choosing the robotic option could have been "purchased" at the rate of $\$ 207,232$ per QALY. This value came from simply dividing the incremental cost by the incremental quality of life gained.

The results of the one-way sensitivity analyses for the probabilities of the various complications are shown in 
Table 3. Surgical procedure costs.

\begin{tabular}{ccc}
\hline & Vaginal mesh hysteropexy & Robotic sacrocolpopexy \\
\hline Variable $_{\text {Mesh device }}^{*}$ & Cost (range) in US \$ & Cost (range) in US \$ \\
Surgeon Payments $^{* *}$ & 1649 & 882 \\
Anesthesiologist Payments $^{t}$ & $2213(1363-2647)$ & $2142(1418-2731)$ \\
Non-physician salary costs $_{\text {Material Costs }}{ }^{*}$ (unrelated to robotics $)$ & $1767(1230-2888)$ & $1830(1410-2788)$ \\
Robot-specific material costs $^{\text {s }}$ & $2539(2283-2909)$ & $3794(3565-4277)$ \\
\hline
\end{tabular}

${ }^{*}$ Actual hospital cost for the Uphold ${ }^{\mathrm{TM}}$ Vaginal Support System or sacrocolpopexy mesh. ${ }^{* *}$ Actual payments to surgeon. ${ }^{\dagger}$ Actual payments to anesthesiologists.

${ }^{¥}$ Actual hospital costs for all disposable and non-disposable materials and drugs used in operating room as well as pre-op holding area and post-anesthesia unit.

${ }^{\S}$ Actual hospital costs for all robot-specific disposable and non-disposable materials \& uterine morcellation device.

Table 1. Focusing on effectiveness (QALYs), there were five threshold values discovered in the sensitivity analyses, but all of these fell outside of clinically plausible ranges. For example, the surgical mortality for both robotic sacrocolpopexy and the Uphold ${ }^{\mathrm{TM}}$ procedure were estimated at 0.0006 . Because it was so rare, the mortality rate of robotic surgery would have had to rise nearly sixty fold (to 0.03541 ) before the overall effectiveness of robotic surgery in the model would have been less than that of the vaginal mesh system based on mortality rates alone. In another example, the estimated rate for cystotomy during robotic surgery would have had to go from 0.013 to 0.746 before the model would have "flipped" to render the Uphold $^{\mathrm{TM}}$ procedure more effective based on cystotomy rates alone (i.e. without changing any other factors in the decision tree).

Only one threshold was produced when calculating CE Ratios-meaning that the Uphold ${ }^{\mathrm{TM}}$ operation was more cost-effective in virtually all scenarios. The estimate for the probability of surgical site infection for the Uphold ${ }^{\mathrm{TM}}$ system was 0.005 . For robotic sacrocolpopexy to have become the more cost-effective approach, the rate of surgical site infection following the vaginal mesh repair would have had to increase to 0.51 , which is not plausible.

The "plausible ranges" used for the sensitivity analyses regarding LUTS and re-operation for prolapse was somewhat narrower due to the relatedness of these variables to each other. Table 4 illustrates the constraints these variables put on each other. We performed two-way and threeway sensitivity analyses to test the robustness of the model for these complications.

Estimates for the dependence of LUTS, de novo chronic pain, and re-operation for prolapse upon each other were derived from the clinical experience of the senior authors (PC and CS). Given the lack of solid empirical evidence in the literature on this topic, special attention was given to these estimates in the sensitivity analyses. None of the expert panel's estimates produced threshold values, even within large ranges. For example, the model was robust concerning the probability of a given robotic sacrocolpopexy patient experiencing a combination of LUTS, de novo chronic pain and re-operation for prolapse. Our clinical estimate of that scenario was 0.10 for the vaginal mesh procedure, and even when that estimate was lowered to 0.005 robotic sacrocolpopexy remained more effective.

Sensitivity analyses on the estimates for quality of life (from the expert panel) produced no thresholds even with very large ranges. Therefore, even marked variation in the effectiveness estimates did not change overall QALYs or CE Ratios enough to reach any thresholds. That is, the model was robust concerning quality of life.

Sensitivity analyses on the costs of the various complications also produced no thresholds, even though the ranges we tested were from $\$ 1$ to extremely high nonplausible amounts (Table 5).

Surgical costs for both procedures, however, did produce threshold values. If the surgical costs for robotic sacrocolpopexy were reduced by $38 \%$ to $\$ 13,560$, then robotic-assisted surgery would become the more costeffective strategy. Likewise, if the surgical costs for the vaginal mesh system increased by $34 \%$ to $\$ 19,900$, then robotic surgery would become the cost-effective strategy.

To test the model for its vulnerability to imprecise inputs, we changed the rates of the most statistically important complications at the same time we varied procedural costs. For example, we reduced the robotic surgical cost estimate to $\$ 16,747$ (23\% decreases) and lowered the rate of re-operation for prolapse to 0.01 , then carried out sensitivity analyses on de novo chronic pain. Varying any two of the outcome probabilities at the same time like this (e.g. LUTS and mesh exposure) failed to produce threshold values with respect to effectiveness or CE Ratio.

\section{DISCUSSION}

Based on the hospital perspective and considering only 
Table 4. Joint probability estimates of LUTS*, de novo chronic pain, and $\mathrm{POP}^{* *}$ recurrence.

\begin{tabular}{ccc}
\hline & \multicolumn{2}{c}{ POP $^{* *}$ Recurrence } \\
\cline { 2 - 3 } & Yes & No \\
\hline LUTS \& Pain & 0.006 & 0.036999957 \\
LUTS Only & 0.006 & 0.001700043 \\
Pain Only & 0.000000002 & 0.000000041 \\
No LUTS \& No Pain & 0.047999998 & 0.901299959 \\
\end{tabular}

*Lower urinary tract symptoms. ${ }^{* *}$ Pelvic Organ Prolapse.

Table 5. Estimates and sensitivity analysis for costs related to complications.

\begin{tabular}{ccc}
\hline Adverse Clinical Outcomes & Estimate (in U.S. \$) & Analysis Range \\
\hline Surgical Mortality & 9077 & $1000-30,000$ \\
Blood Transfusion & 892 & $1-20,000$ \\
Cystotomy & 125 & $1-10,000$ \\
Surgical Site Infection & 9876 & $1-30,000$ \\
Mesh Exposure (Office) & 1 & $1-10,000$ \\
Mesh Exposure (Surgery) & 9876 & $1-30,000$ \\
de novo LUTS & 267 & $1-4000$ \\
de novo Chronic Pain & 9876 & $1-20,000$ \\
Reoperation for Prolapse & 12,967 & $1-30,000$ \\
\hline
\end{tabular}

*Lower urinary tract symptoms.

the first 12 months after surgery, the Uphold ${ }^{\mathrm{TM}}$ Vaginal Support System was more cost-effective than robotic supracervical hysterectomy/sacrocolpopexy no matter how we varied our estimates for probabilities and quality of life. The only variable in our model that could be changed to result in an opposite conclusion was surgical cost. Even then, the cost savings required for robotic surgery to be considered the more cost-effective option was greater than $30 \%$. Moreover, we did not even consider the costs of the robot itself. These results held up to all sensitivity analyses - meaning that no clinically plausible changes in any of the other variables in our model resulted in a different decision concerning the final costeffectiveness. The fact that varying the costs of complications within the model did not produce any threshold values was primarily due to the rarity of most complications. As such, extremely low or high costs for any one complication did not tend to overwhelm the costs for the entire model. When no single dominant complication exists in a model like ours, the resultant "final answer" of the decision tree is considered robust (as ours was). From a societal perspective, the question transitions to opportunity costs. Would it be wiser to spend that incremental cost on another health care opportunity? To answer that question, one must consider QoL estimates.

Robotic-assisted laparoscopic sacrocolpopexy afforded the individual patient, on average, a higher QoL than did the vaginal mesh system ( 0.9645 vs. 0.9309 QALYs, respectively). This result begs the question: is the difference of 0.0336 QALY in the first year post procedure worth the $\$ 6,963$ in incremental costs? If so, who should pay those costs?

From the societal perspective, 0.9309 QALYs in the first year post procedure can be purchased via the Uphold $^{\text {TM }}$ system at a rate of $\$ 15,995$ per QALY (the CE Ratio). The additional 0.0336 QALYs that could be purchased with robotic-assisted sacrocolpopexy would be at a rate of $\$ 207,232$ per QALY (the Incremental CE Ratio). The benchmark value used by cost-effectiveness studies in the U.S. is $\$ 50,000$ per QALY [15]. Therefore even when the cost of the robot itself was not considered, the Uphold $^{\mathrm{TM}}$ approach was far more cost-effective.

As is often the case when one procedure is both more effective and more costly, the Incremental CE Ratio is markedly higher than the CE Ratio of the less expensive procedure. From a societal perspective, directly compar- 
ing $\$ 15,995$ (the vaginal mesh CE Ratio) with $\$ 207,232$ (the Incremental CE Ratio) suggests that one could get almost as good an outcome with the less expensive procedure and that paying for the more effective procedure sends one up a slippery slope of escalating costs.

Our study design had several obvious limitations. First and foremost, our results should only be viewed from the cost perspective we chose: the hospital perspective. We chose this perspective because doing so allowed us to compare actual cost differences between the two surgical procedures. Some would argue that choosing a societal cost perspective would have been more appropriate, but doing so would have injected far more cost subjectivity into the model. Also, in order to make the decision tree a manageable size, we chose to limit the number of variations we considered within each clinical outcome. For example, we made the assumption that blood transfusions would happen without further ramifications. Obviously this assumption would not hold up in all cases, but mathematically speaking, inclusion of all possible transfusion complications would not have been worth the exponential tree growth that would have resulted from doing so. In other words, inclusion of other more rare complications would not have resulted in a different final outcome. In this regard, decision analytic studies come closer to modeling the kind of decision making that physicians carry out on a daily basis. For example, on some level pelvic surgeons know that the possibility of complications from blood transfusions always exists, but we don't tend to let the possibility of complications from those rare blood transfusions drive our treatment choices.

In addition, we chose not to consider the cost of the robotic hardware or its upkeep (nearly \$2 million in some cases). We made this choice because of the complex accounting systems for consideration of depreciation. The physical age of a given robot determines its value at any given point in time. Furthermore, many robots in place around the country (ours included) were donated to their hospitals. Therefore, we felt that it would be impossible to come up with a fair "average" cost for the robotic hardware.

As is true for any decision analytic model, our output could only be as good as the model inputs. Although we used estimates from what we considered the best studies in the field for almost all of the inputs, we had to use our clinical experience to provide prevalence estimates for complications that were not specifically addressed in any prior studies. However, our use of sensitivity analysis surely mitigated this limitation. By listing the plausible range for each point estimate, we give the reader an opportunity to scrutinize each of these assumptions. To the extent that our plausible ranges can hold up to such scrutiny, the sensitivity analyses speak to the robustness of our results. That is, arguments about the validity of certain point estimates are mute as long as those point estimates existed within the range of values we tested in the model.

Finally, our results are not relevant beyond the scope of our basic research question - namely whether the Uphold $^{\mathrm{TM}}$ procedure or the robotic sacrocolpopexy offer any cost/ QoL advantages among a group of women offered a choice between the two. One cannot apply these results to any other groups of POP patients or any other transvaginal mesh procedures.

\section{CONCLUSION}

Notwithstanding these limitations, we found the Uphold $^{\mathrm{TM}}$ system to be more cost-effective than roboticassisted laparoscopic supracervical hysterectomy and sacrocolpopexy.

\section{ACKNOWLEDGMENTS}

Study Funding provided by an unrestricted educational grant from Boston Scientific, Natick, MA.

\section{DISCLOSURES}

Dr. Salamon is a paid consultant for American Medical Systems.

Both Dr. Salamon and Dr. Culligan are paid consultants and instructors for Intuitive Surgical.

None of the other authors have any potential conflicts of interest to disclose.

\section{REFERENCES}

[1] FDA Update (2011) Urogynecologic surgical mesh: Update on the safety and effectiveness of transvaginal placement for pelvic organ prolapse.

[2] Parker, W.H., Broder, M.S., Liu, Z., Shoupe, D., Farquhar, C., et al. (2005) Ovarian conservation at the time of hysterectomy for benign disease. Obstetrics \& Gynecology, 106, 219-226. http://dx.doi.org/10.1097/01.AOG.0000167394.38215.56

[3] Culligan, J., Murphy, M., Blackwell, L., Hammons, G., Graham, C., et al. (2002) Long-term success of abdominal sacral colpopexy using synthetic mesh. American Journal of Obstetrics \& Gynecology, 187, 1473-1482. http://dx.doi.org/10.1067/mob.2002.129160

[4] Shull, B., Capen, C., Riggs, M. and Kuehl, T. (1992) Preoperative and postoperative analysis of site-specific pelvic support defects in 81 women treated with sacrospinous ligament suspension and pelvic reconstruction. American Journal of Obstetrics \& Gynecology, 166, 1764-1768. http://dx.doi.org/10.1016/0002-9378(92)91567-T

[5] Morley, G. and DeLancey, J. (1990) Sacrospinous ligament fixation for eversion of the vagina. American Journal of Obstetrics \& Gynecology, 162, 295-296. http://dx.doi.org/10.1016/0002-9378(90)90881-7

[6] Estrade, J., Agostini, A., Roger, V., Dallay, D., Blanc, B., 
et al. (2004) Sacrospinous colpopexy complications. $G y$ necologie Obstetrique \& Fertilite, 32, 850-854.

[7] Geller, E., Siddiqui, N., Wu, J. and Visco, A. (2008) Short- term outcomes of robotic sacrocolpopexy compared with abdominal sacrocolpopexy. Obstetrics \& Gynecology, 112, 1201-1206.

http://dx.doi.org/10.1097/AOG.0b013e31818ce394

[8] Hefni, M. and El-Toukhy, T. (2006) Long-term outcome of vaginal sacrospinous colpopexy for marked uterovaginal prolapse. European Journal of Obstetrics \& Gynecology and Reproductive Biology, 127, 257-263. http://dx.doi.org/10.1016/j.ejogrb.2005.11.028

[9] Tayrac, R., Boileau, L., Fara, J., Monneins, F., Raini, C., et al. (2010) Bilateral anterior sacrospinous ligament suspension associated with a paravaginal repair with mesh: Short-term clinical results of a pilot study. International Urogynecology Journal and Pelvic Floor Dysfunction, 21, 293-298. http://dx.doi.org/10.1007/s00192-009-1036-1

[10] Boyd, L., Novetsky, A. and Curtin, J. (2010) Effect of surgical volume on route of hysterectomy and short-term morbidity. Obstetrics \& Gynecology, 116, 909-915. http://dx.doi.org/10.1097/AOG.0b013e3181f395d9

[11] Culligan, P., Littman, P., Salamon, C., Priestley, J. and Shariati, A. (2010) Evaluation of a transvaginal mesh de- livery system for the correction of pelvic organ prolapse: Subjective and objective findings at least 1 year after surgery. American Journal of Obstetrics \& Gynecology, 203, E1-E6.

[12] Nygaard, I., McCreery, R., Brubaker, L., Connolly, A., Cundiff, G., et al. (2004) Abdominal sacrocolpopelxy: A comprehensive review. Obstetrics \& Gynecology, 104, 805-823. http://dx.doi.org/10.1097/01.AOG.0000139514.90897.07

[13] Withagen, M., Milani, A., Boon, J., Vervest, H. and Vierhout, M. (2011) Trocar-guided mesh compared with conventional vaginal repair in recurrent prolapse. Obstetrics \& Gynecology, 117, 242-250.

[14] Granese, R., Candiani, M., Perino, A., Romano, F. and Cucinella, G. (2009) Laparoscopic sacrocolpopexy in the treatment of vaginal vault prolapse: 8 years experience. European Journal of Obstetrics \& Gynecology and Reproductive Biology, 146, 227-231. http://dx.doi.org/10.1016/j.ejogrb.2009.06.013

[15] Neumann, P., Sandberg, E., Bell, C., Stone, P. and Chapman, R. (2000) Are pharmaceuticals cost-effective? A review of the evidence. Health Affairs, 19, 92-109. http://dx.doi.org/10.1377/hlthaff.19.2.92 